\title{
Nonlinear Effects with Ultrashort Laser Pulses
}

\author{
P. WasylczyK ${ }^{*}$, W. Wasilewski, M. Trippenbach \\ AND C. RADZEWICZ \\ Institute of Experimental Physics, Warsaw University \\ Hoża 69, 00-681 Warsaw, Poland
}

\begin{abstract}
Propagation of an intense femtosecond laser pulse through a transparent nonlinear medium such as dielectric leads to a number of phenomena. In our experiment we observed complex spatial, spectral, and temporal structures appearing in the initially smooth femtosecond laser pulse when the pulse power is comparable to or higher than the critical power for self-focusing. We have also developed a complete, 3-dimensional theoretical model to describe the observed phenomena.
\end{abstract}

PACS numbers: 42.65.Jx, 42.65.Sf

\section{Introduction}

Although the chase for records in the world-shortest pulse is still open, there is already a number of well established and developed techniques in the ultrafast laser technology that have opened a possibility for applications outside research laboratories. For example, in multiphoton microscopy, femtosecond laser pulses with high instantaneous power are used to excite multiphoton fluorescence in the sample leading to increased spatial resolution. New applications of femtosecond laser pulses are also continuously developed. In recent proposals by two groups $[1,2]$ extremely broad band femtosecond pulses are used to generate frequency combs that enable direct comparison of optical frequencies with the cesium frequency standard. This technique could, in principle, enable construction of a new generation of frequency standards with accuracy of the order of $10^{-17}$. There are, in principle, two reasons why people are interested in studying nature with ultrashort pulses. On the one hand, one can benefit from pulse duration - the

*corresponding author; e-mail: pwasylcz@fuw.edu.pl 
shorter the pulses available the faster the processes that can be studied with sufficient temporal resolution. This includes, among others, such phenomena as early stages of chemical reactions [3-5], energy transfer in molecules [6-8], carrier dynamics in semiconductors [9-11]. The other advantage lies in power - even a pulse with a moderate energy, if short enough, offers powers in the ranges much higher than available from any other source [12]. For example, in the terawatt region the field-atom interaction is far from what we expected from our low-energy based experiences.

Experiments with femtosecond laser pulses in biophysics or physical chemistry are truly fascinating but here we will focus on a much simpler case. The simplest experiment that one could think about deals with propagation of such pulses through a transparent medium (transparent means here that we are far from any resonances and thus the medium response is practically instantaneous). And, as propagation is an intrinsic property of electromagnetic waves, this experiment is performed every time one uses a femtosecond laser in the laboratory. Large band width of ultrashort light pulses causes that whenever they propagate through a dispersive medium their shape changes even in a low intensity regime when only linear response of the medium is relevant. This can lead to distortion of a pulse focused with a lens [13] or pulse tilting in birefringent media [14]. The problem gets a bit more complicated when higher intensities are used. As we have already mentioned, very short pulses easily provide high instantaneous intensities and therefore nonlinear response of the medium cannot be neglected. Since we are interested in isotropic media, the lowest nonlinearity to be considered is of the 3rd order. Thus, to analyze pulse propagation in such a case we have to take into consideration diffraction, dispersion, as well as spatial and temporal processes resulting from the third-order susceptibility. Propagation of femtosecond laser pulses in such regime has been studied before [15-17]. It was found that, contrary to the long pulse case, there is no catastrophic self-focusing when short $(<100 \mathrm{fs}$ ) pulses are used with powers of the order of the critical power (that equals $2.6 \mathrm{MW}$ for fused silica at $800 \mathrm{~nm}$ ). Self-focusing is avoided because of pulse splitting; a combined action of dispersion and nonlinearity leads to a break-up of the pulse into two, or more, subpulses in time domain. All the previous studies have been limited to the measurements on the central part of the beam close to the beam axis. In this contribution we extend these studies to the peripheral parts of the beam. We also show that pulse splitting strongly depends on the beam size.

\section{Theoretical approach}

We have derived a new formulation for propagating optical pulses and $\mathrm{cw}$ beams, valid to all orders in the dimensionless variables $\eta=\left(\omega_{0} / \tau_{0}\right)^{-1}$ and $\epsilon=\left(k_{0} \sigma_{0}\right)^{-1}$. Here $\tau_{0}$ is the pulse duration, $\omega_{0}$ is the central frequency of the pulse, $k_{0}=n_{0} \omega_{0} / c$ is central wave vector magnitude in the medium, $\sigma_{0}$ is the 
transverse pulse width and $n_{0}=n\left(\omega_{0}\right)$ is the refractive index at the central frequency. This method can be applied to pulse propagation in both linear and nonlinear regimes. The application to the linear regime has been described in [18-20] and was experimentally verified [14]. The method can be applied to propagate extremely short pulses and extremely focused pulses and cw beams without approximation. Consequently, linear near-field diffraction effects can be exactly calculated in dispersive media (even non-isotropic dispersive media [19]). Here we extend the approach of Ref. [19], which is based on a consistent and mathematically rigorous expansion of the linear dispersion relation, to include a nonlinear optical response of the medium. Previous studies have treated nonlinearity only to lowest orders [21-25, 15]. To go beyond these lowest-order approximations in the nonlinearity and yet retain all orders in $\eta$ and $\epsilon$, we generalize our method by incorporating a full perturbation analysis developed by Fibich $[26,27]$ for $\mathrm{cw}$ beams into the formulation previously used for linear pulse propagation [20]. Our treatment is limited only by the assumption that the coupling of backscattering modes can be neglected; we consider only a one-way propagation of the field and do not the two-way propagation boundary matching problem [28]. The present work is the first attempt to compare our expansion with experimental results for propagation of a very short and strongly focused pulse in anisotropic Kerr-type nonlinear medium.

We begin by considering the wave equation for the electric field

$$
\left(\nabla_{\perp}^{2}+\frac{\partial^{2}}{\partial z^{2}}-\frac{1}{c^{2}} \frac{\partial^{2}}{\partial t^{2}}\right) \boldsymbol{E}(\boldsymbol{x}, t)=\frac{4 \pi}{c^{2}} \frac{\partial^{2}}{\partial t^{2}}\left[\boldsymbol{P}^{\mathrm{L}}(\boldsymbol{x}, t)+\boldsymbol{P}^{\mathrm{NL}}(\boldsymbol{x}, t)\right],
$$

where $\boldsymbol{P}^{\mathrm{L}}$ and $\boldsymbol{P}^{\mathrm{NL}}$ are the linear and nonlinear polarization vectors respectively, or, in Fourier space,

$$
\left(k_{x}^{2}+k_{y}^{2}+k_{z}^{2}-\frac{1}{c^{2}} \omega^{2}\right) \boldsymbol{E}(k, \omega)=\frac{4 \pi}{c^{2}} \omega^{2}\left[\boldsymbol{P}^{\mathrm{L}}(\boldsymbol{k}, \omega)+\boldsymbol{P}^{\mathrm{NL}}(\boldsymbol{k}, \omega)\right] .
$$

We consider the case of a linear isotropic medium where

$$
\boldsymbol{P}^{\mathrm{L}}=\frac{1}{(2 \pi)^{2}} \int \mathrm{d}^{3} k \mathrm{~d} \omega \chi^{(1)}(\boldsymbol{k}, \omega) \boldsymbol{E}(\boldsymbol{k}, \omega) \exp (\mathrm{i} \boldsymbol{k} \cdot \boldsymbol{x}-\mathrm{i} \omega t)
$$

and

$$
\chi^{(1)}(\boldsymbol{k}, \omega)=\frac{\epsilon(\boldsymbol{k}, \omega)-1}{4 \pi}=\frac{n^{2}(\omega)-1}{4 \pi} .
$$

At first we set the nonlinear polarization term to zero. Let us assume that the electric field and induced polarization are linearly polarized along the $x$-axis. Next we rewrite the propagation equation in terms of the slowly varying envelope (SVE) $A$ of the electric field for a light pulse, $\boldsymbol{E}(\boldsymbol{x}, t)=A(\boldsymbol{x}, t) \exp \left(\mathrm{i} k_{0} \cdot \boldsymbol{x}-\mathrm{i} \omega_{0} t\right) \hat{\boldsymbol{x}}$, or in Fourier space, $E_{x}(\omega, k)=A\left(\omega-\omega_{0}, k-k_{0}\right)$. The SVE multiplies the quickly oscillating temporal and spatial terms associated with central frequency $\omega_{0}$ and central wave vector $\boldsymbol{k}_{0}=k_{0} \hat{z}=n_{0}\left(\omega_{0} / c\right) \hat{z}\left(n_{0}=n\left(\omega_{0}\right)\right)$ in the expression for the 
electric field. Note that we have chosen the $z$-axis to be along the central wave vector. Substituting the SVE into Eq. (2), changing variables $\omega \rightarrow \omega+\omega_{0}, k \rightarrow$ $k+k_{0}$ so as to remove the central frequency and central wave vector from the SVE, Eq. (2) yields

$$
\left[k_{x}^{2}+k_{y}^{2}+\left(k_{z}+k_{0}\right)^{2}-\left(\frac{n\left(\omega+\omega_{0}\right)\left(\omega+\omega_{0}\right)}{c^{2}}\right)^{2}\right] \boldsymbol{A}(\boldsymbol{k}, \omega)=0 .
$$

We can now use an inverse Fourier transform and write Eq. (1) in terms of the SVE as

$$
\begin{aligned}
&\left(\nabla_{\perp}^{2}\right.\left.+2 \mathrm{i} k_{0} \frac{\partial}{\partial z}+\frac{\partial^{2}}{\partial z^{2}}\right) A(\boldsymbol{x}, t)=\frac{-1}{(2 \pi)^{2} c^{2}} \int \mathrm{d}^{3} k \mathrm{~d} \omega \\
& \quad \times\left[\left(\omega+\omega_{0}\right)^{2} n^{2}\left(\omega+\omega_{0}\right)-\omega_{0}^{2} n_{0}^{2}\right] A(\boldsymbol{k}, \boldsymbol{\omega}) e^{(\mathrm{i} \boldsymbol{k} \cdot \boldsymbol{x}-\mathrm{i} \omega t)} .
\end{aligned}
$$

Equation (4) can be rearranged to take the form

$$
\frac{\partial}{\partial z} A=\frac{1}{2 i k_{0}}\left(-\nabla_{\perp}^{2}-k_{0}^{2} \mathcal{F}-\frac{\partial^{2}}{\partial z^{2}}\right) A,
$$

where the right hand side (RHS) of Eq. (4) has been written as $-k_{0}^{2} \mathcal{F} A$ thereby defining the linear differential operator $\mathcal{F}$, which can be obtained as a power series of $\partial / \partial t$ by expanding integrand of the RHS of $\mathrm{Eq}$. (4) in $\omega$ about $\omega_{0}$, and evaluating Fourier transform.

Our expansion takes a particularly enlightening form in dimensionless units in which time is measured in units of temporal pulse duration $\tau_{\mathrm{p}}$, length in the transverse directions is in units of the transverse pulse width $\sigma_{\mathrm{p}}$, and length in the propagation direction $z$ is measured in diffraction length $L_{\mathrm{DF}}=k_{0} \sigma_{\mathrm{p}}^{2}[25,26]$. The final expression of the propagation equation will be given in terms of the two dimensionless parameters $\epsilon=\left(k_{0} \sigma_{p}\right)^{-1}$ and $\eta=\left(\omega_{0} \tau_{p}\right)^{-1}$.

Equation (4) in dimensionless units of $x, y, z$, and $t$ may be written as

$$
\left(L_{\mathrm{DF}}^{-1} \frac{\partial}{\partial z}\right) A=\frac{\mathrm{i} k_{0}}{2}\left[\mathcal{L}_{0}+\frac{1}{k_{0}^{2}}\left(L_{\mathrm{DF}}^{-1} \frac{\partial}{\partial z}\right)^{2}\right] A
$$

where the operator $\mathcal{L}$, is defined as $\mathcal{L}_{0} \equiv \epsilon^{2} \nabla_{\perp}^{2}+\mathcal{F}\left(\omega_{0} \eta \frac{\partial}{\partial t}\right)$. This equation can be solved exactly to give

$$
\mathcal{O}_{0} A=i k_{0}\left(\sqrt{1+\mathcal{L}_{0}}-1\right) A
$$

It can be easily verified that Eq. (6) yields the original wave equation by adding $\mathrm{i} k_{0} A$ to both sides of $(6)$ and squaring the resulting equation. Operator $\mathcal{O}_{0}=\mathrm{i} k_{0}(\sqrt{1+\mathcal{L}}-1)$ in Eq. (6) can be expanded in the Taylor series in $\epsilon$ and $\eta$ (in spatial and temporal derivatives) [29].

We now add nonlinear polarization and for that purpose we consider a nonlinear medium with a Kerr-type nonlinear susceptibility of the form 
$\chi_{i j k l}^{(3)}\left(-\omega ; \omega_{1},-\omega_{2}, \omega_{3}\right)$. The nonlinear source term in the wave equation can be written in terms of the SVE as follows:

$$
\begin{aligned}
& \frac{4 \pi}{c^{2}} \frac{\partial^{2}}{\partial t^{2}} P_{i}^{\mathrm{NL}}(\boldsymbol{x}, t)=\frac{-4 \pi}{c^{2}(2 \pi)^{8}} \int \mathrm{d}^{3} k \mathrm{~d}_{\omega} \mathrm{d}^{3} k_{1} \mathrm{~d} \omega_{1} \mathrm{~d}^{3} k_{2} \mathrm{~d} \omega_{2} \mathrm{~d}^{3} k_{3} \mathrm{~d} \omega_{3} \\
& \quad \times \delta\left(k-k_{1}+k_{2}-k_{3}\right) \delta\left(\omega-\omega_{1}+\omega_{2}-\omega_{3}\right) \mathrm{e}^{\left[\mathrm{i}\left(\boldsymbol{k}+\boldsymbol{k}_{0}\right) \cdot \boldsymbol{x}-\mathrm{i}\left(\omega+\omega_{0}\right) t\right]} \\
& \quad \times\left(\omega_{0}+\omega\right)^{2} \chi_{i j k l}^{(3)}\left[-\left(\omega_{0}+\omega\right) ; \omega_{0}+\omega_{1},-\left(\omega_{0}+\omega_{2}\right), \omega_{0}+\omega_{3}\right] \\
& \quad \times A_{j}\left(\boldsymbol{k}_{1}, \omega_{1}\right) A_{k}^{*}\left(\boldsymbol{k}_{2}, \omega_{2}\right) A_{l}\left(\boldsymbol{k}_{3}, \omega_{3}\right) .
\end{aligned}
$$

Here we have assumed that the wave vector dependence of the nonlinear susceptibility is unimportant. If one neglects the frequency dependence of $\chi_{i j k l}^{(3)}$, then in the coordinate space one finds

$$
\begin{aligned}
& \frac{4 \pi}{c^{2}} \frac{\partial^{2}}{\partial t^{2}} P_{i}^{\mathrm{NL}}(\boldsymbol{x}, t)=-\frac{4 \pi \chi^{(3)}}{c^{2}} \\
& \quad \times\left[\omega_{0}^{2}|A|^{2} A+2 \omega_{0} \frac{\partial\left(|A|^{2} A\right)}{\partial t}+\frac{\partial^{2}\left(|A|^{2} A\right)}{\partial t^{2}}\right] \mathrm{e}^{\mathrm{i}\left(k_{0} z-\omega_{0} t\right)}
\end{aligned}
$$

Note the presence of the two time derivative terms on the RHS of the equation. The second term on the RHS gives rise to self-steepening and the third is a higher-order term.

In order to develop an expansion of equation of motion for a light pulse to all orders in $\eta$ and $\epsilon$ that is valid for nonlinear pulse propagation, we now introduce (in dimensionless units) a new operator $\mathcal{L}$ to replace the $\mathcal{L}_{0}$ :

$$
\mathcal{L} A \equiv \mathcal{L}_{0} A+\frac{4 \pi \chi^{(3)}}{n_{0}^{2}}\left[|A|^{2} A+2 \eta \frac{\partial\left(|A|^{2} A\right)}{\partial t}+\eta^{2} \frac{\partial^{2}\left(|A|^{2} A\right)}{\partial t^{2}}\right]
$$

Note that $\mathcal{L}$ is $z$ dependent through $A(x, y, z, t)$, and does not commute with $\partial / \partial z$.

With the new $\mathcal{L} A$ we cannot obtain as simple result as Eq. (6), valid for the linear propagation of optical pulses, but we still obtain a perturbative expansion in the parameters $\epsilon$ and $\eta$. Equation (5) is now modified and becomes

$$
\mathcal{O} A \equiv\left(L_{\mathrm{DF}}^{-1} \frac{\partial}{\partial z}\right) A=\frac{\mathrm{i} k_{0}}{2}\left[\mathcal{L}+\frac{1}{k_{0}^{2}}\left(L_{\mathrm{DF}}^{-1} \frac{\partial}{\partial z}\right)^{2}\right] A=\frac{\mathrm{i} k_{0}}{2}\left(\mathcal{L}+\frac{1}{k_{0}^{2}} \mathcal{O}^{2}\right) A
$$

If we operate on Eq. (10) with $\mathcal{O}$ and divide the resulting equation by $k_{0}^{2}$ we find

$$
\frac{1}{k_{0}^{2}} \mathcal{O}^{2} A=\left(\frac{\mathrm{i}}{2} \epsilon^{2} \frac{\partial[\mathcal{L} A(z)]}{\partial z}-\mathrm{O}\left(\epsilon^{4}\right)\right)
$$

Hence, the RHS of Eq. (11) contains both $\partial A / \partial z$ and $\partial A^{*} / \partial z$ terms. To carry out the expansion we simply replace $\partial A / \partial z$ with the right hand side of Eq. (10) and $\partial A^{*} / \partial z$ with the RHS of the complex conjugate of Eq. (10). Next we isolate terms of different order in $\epsilon$. This procedure is tedious due to the large number of terms that must be included, but it is mathematically rigorous. Expansion can be carried 
out using the symbolic mathematics program Maple [29], and after carrying out the algebra for our case of an isotropic medium we finally obtain

$$
\begin{aligned}
& L_{\mathrm{DF}}^{-1} \frac{\partial A}{\partial z}=-\beta_{1} \omega_{0} \eta \frac{\partial A}{\partial t}-\frac{\mathrm{i} \beta_{2} \omega_{0}^{2} \eta^{2}}{2} \frac{\partial^{2} A}{\partial t^{2}}+\frac{\beta_{3} \omega_{0}^{3} \eta^{3}}{6} \frac{\partial^{3} A}{\partial t^{3}}+\frac{\mathrm{i} \beta_{4} \omega_{0}^{4} \eta^{4}}{24} \frac{\partial^{4} A}{\partial t^{4}} \\
& +\mathrm{i} \frac{\gamma_{x x} k_{0}^{2} \epsilon^{2}}{2}\left(\frac{\partial^{2} A}{\partial x^{2}}+\frac{\partial^{2} A}{\partial y^{2}}\right)+\frac{\gamma_{t x x} \omega_{0} k_{0}^{2} \epsilon^{2} \eta}{2}\left(\frac{\partial^{3} A}{\partial x^{2} \partial t}+\frac{\partial^{3} A}{\partial y^{2} \partial t}\right) \\
& +\mathrm{i} \frac{\gamma_{x x x x} k_{0}^{4} \epsilon^{4}}{8}\left(\frac{\partial^{4} A}{\partial x^{4}}+2 \frac{\partial^{4} A}{\partial x^{2} \partial y^{2}}+\frac{\partial^{4} A}{\partial y^{4}}\right)+\ldots+\frac{2 \pi \chi^{(3)} \omega_{0}}{n c} \\
& \quad \times\left\{\mathrm{i}|A|^{2} A+\eta\left[\left(\frac{c \beta_{1}}{n}-2\right) \frac{\partial\left(|A|^{2} A\right)}{\partial t}\right]-\mathrm{i} \epsilon^{2} \frac{1}{n^{2}}\left[A \frac{\partial A^{*}}{\partial x} \frac{\partial A}{\partial x}+A \frac{\partial A^{*}}{\partial y} \frac{\partial A}{\partial y}\right.\right. \\
& \left.\left.\quad+\frac{1}{2} A^{*}\left(\frac{\partial A}{\partial x}\right)^{2}+\frac{1}{2} A^{*}\left(\frac{\partial A}{\partial y}\right)^{2}+|A|^{2} \frac{\partial^{2} A}{\partial x^{2}}+|A|^{2} \frac{\partial^{2} A}{\partial y^{2}}\right]\right\} .
\end{aligned}
$$

We have only included the first-order time-derivative nonlinear terms and we have not included higher-order terms in $\epsilon$ in Eq. (12). We can, however, easily obtain higher-order time-derivative and spatial-derivative nonlinear terms and higher-order terms to any desired order in $\epsilon$. When all time-derivative terms are set to zero, we recover the results of Fibich et al. for cw beams [27, 28]. When the nonlinear coupling terms are set to zero, we trivially recover the linear pulse propagation results [20]. Extensive studies of these results will be presented elsewhere [29].

We numerically solve Eq. (12) using split step operator method [30]. The right hand side of Eq. (12) is a sum of two kinds of operators: one diagonal in Fourier domain and the other easily evaluated in space domain. Therefore in each numerical step we solve our equation ignoring all the operators of the first kind, then perform FFT of $A$ and solve it using operators of the second kind only. This method was shown to lead to inaccuracy proportional to $\Delta z^{2}$ only. In fact it is fairly easy to increase the accuracy up to $\Delta z^{3}$, which we actually do as was discussed elsewhere [30]. For computational simplicity we solve our problem in $(2+1) \mathrm{D}$ i.e. we neglect $y$-dependence of the field.

\section{Experiment}

As a source of femtosecond pulses we used a laser system presented in Fig. 1. The output beam from a home-built Ti:sapphire femtosecond oscillator pumped by all-lines argon-ion laser (Coherent Innova, $6 \mathrm{~W}$ ) was used as a seed for the regenerative amplifier (Coherent $\alpha$-1000) pumped by a frequency-doubled Nd:YLF (Coherent 621-D). The pulses from the amplifier were approximately $70 \mathrm{fs}$ long and centered at $800 \mathrm{~nm}$. The pulse energy was $1 \mathrm{~mJ}$ and the repetition rate was $1 \mathrm{kHz}$. We used a home-built FROG apparatus [31, 32] to measure temporal properties of the pulses. The measured amplitude and phase of the pulses in time and frequency 


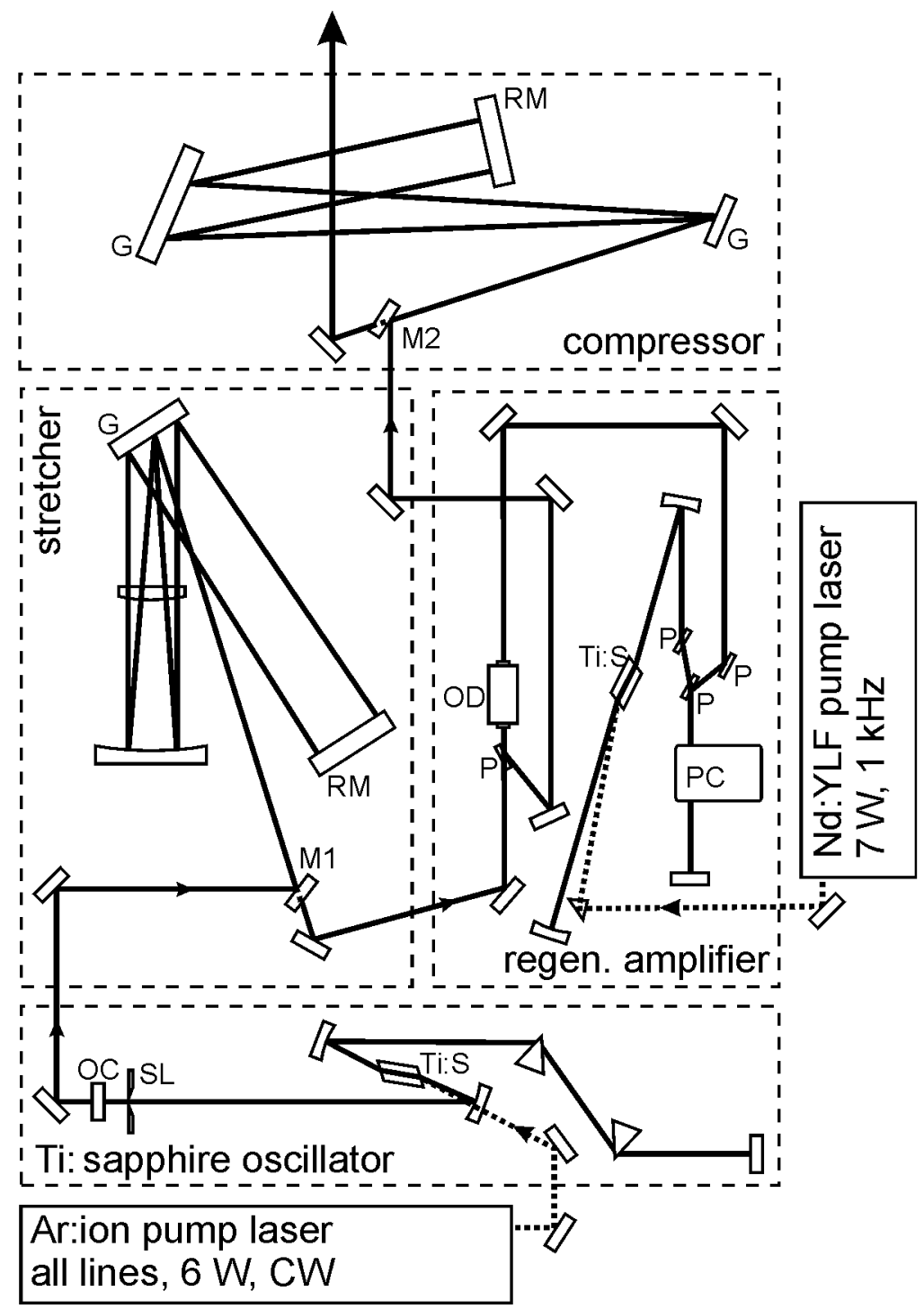

Fig. 1. The laser system used to generate femtosecond pulses. A commercial amplifier system consisting of a stretcher, regenerative amplifier, and compressor is seeded with pulses from a home-built Ti:sapphire oscillator. Ti:S - titanium-doped sapphire crystal, SL - adjustable slit, OC - output coupler, $\mathrm{P}$ - polarizers, OD - optical diode, PC Pockels cell, RM - roof mirror arrangement, G - 1200 grooves $/ \mathrm{mm}$ gratings. Mirrors M1, M2 are placed above beam plane; all steering mirrors are broadband dielectric.

domains are presented in Fig. 2. As is clear from Fig. 2 the pulses from our amplifier were not transform-limited (time-band width product of approximately 0.6 ), as 

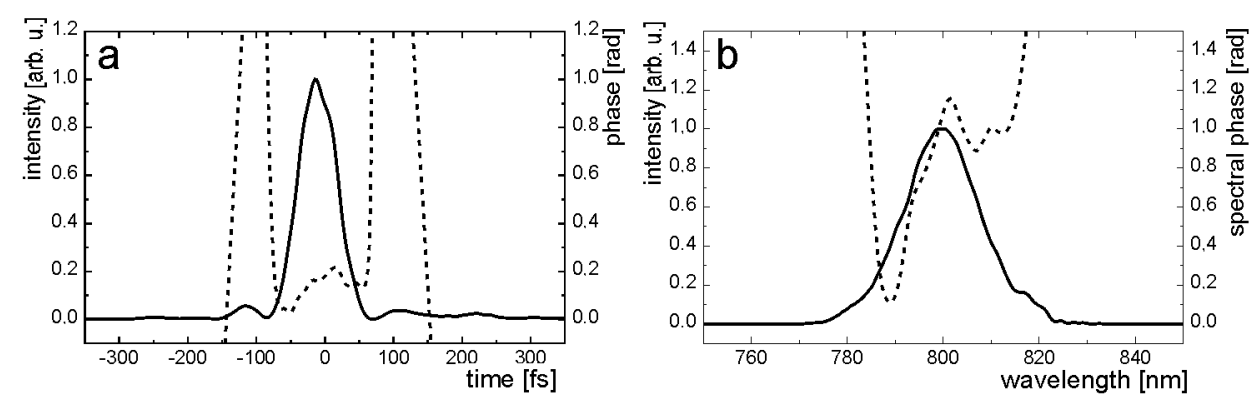

Fig. 2. Laser pulse amplitude and phase in time (a) and frequency (b) domain. The amplitude and phase are marked by continuous and broken lines respectively.

indicated by a nontrivial shape of the temporal phase. This might be considered a drawback of the setup. Actually, it is not really the case. Since we use a numerical code to simulate the pulse propagation, we do not need a well behaving pulse with a smooth amplitude and phase which could be well approximated by analytic expressions. What is really important is that we are able to measure the electric field of the laser pulse and supply this information to the numerical procedure modeling the propagation.

There are three main parts of the experimental setup shown in Fig. 3. The first one was used to condition the laser beam, i.e. to control its spatial properties and pulse energy. Control over spatial properties of the beam is crucial because the spatial distribution influences the propagation. To be able to compare the experimental results with the numerical modeling we have to make sure that we control the spatial properties of the beam. In addition, for efficiency reasons, the numerical code we use is two-dimensional. It is an approximation which holds only

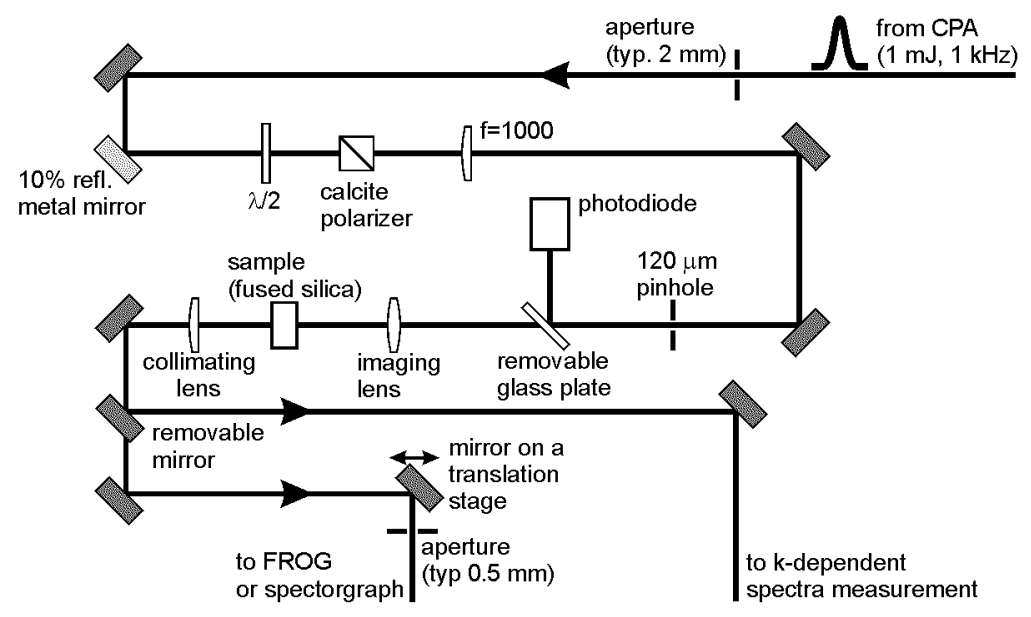

Fig. 3. Experimental setup. See text for details. 
when the laser beam has a perfect cylindrical symmetry. First, the beam passed through an aperture with a diameter of $2 \mathrm{~mm}$. This reduced the residual spread of spectral components in the beam transversal profile (spatial chirp) that might have been introduced in the stretcher-compressor system.

The second stage of the filtering consisted of a $1000 \mathrm{~mm}$ focal length fused silica lens and an interchangeable pinhole (typically $120 \mu \mathrm{m}$ diameter) placed in the focus of the beam. These two elements formed a spatial filter for the wave vectors present in the pulse. The conditions were set in such a way that, to a very good approximation, the far field distribution in the beam behind the pinhole was described by the Airy function. The third stage was responsible for energy control. With a $1 \mathrm{~mJ}$ pulse focused to several tens of micrometers any optical material would be easily damaged. The energies we are interested in are, at least, two orders of magnitude lower. We also would like to have a continuous energy adjustment. A combination of a zero-order half-wave plate and a calcite polarizer was used for this purpose. The pulse energy was monitored with a photodiode and a digitizing oscilloscope. The second part of the experimental setup contained the sample and two fused silica lenses. The first lens was used to image the pinhole on the input face of the sample which was a plane-parallel fused silica plate $7.5 \mathrm{~mm}$ thick. Using imaging lenses with different focal lengths we could vary the diameter of the beam waist while keeping the position of the waist exactly at the input face of the sample. In this contribution we will report the results for two cases: "large beam" and "small beam" which are markedly different. The "large beam" indicates a focus of approximately $120 \mu \mathrm{m}$ diameter, while the "small beam" means a focus of approximately $20 \mu \mathrm{m}$ diameter. The beam behind the sample was collimated with the second lens of $15 \mathrm{~cm}$ focal length and directed towards the diagnostics part of the setup. Since the focal length of this lens is much larger, the Rayleigh range of the beam, the field distribution in the collimated beam corresponded to the far field pattern.

The third part of the setup contained the diagnostic tools. Two types of measurements were performed on the output pulses. Firstly, we could measure spectra of the pulses with an imaging spectrograph equipped with a two-dimensional CCD array (Acton Research Spectra Pro 150, SpectruMM 128 HF). By illuminating the entrance slit of the spectrograph with the collimated output beam we could record two-dimensional maps in the $\omega-k_{\perp}$ space. The maps show the intensity of the output beam as a function of the optical frequency $\omega$ (or wavelength) and the component of the wave vector perpendicular to the propagation direction $k_{\perp}$. Using an adjustable iris pinhole we could also select a part of the beam (approximately $5-10 \%$ ) and direct it into the FROG apparatus to determine the electrical field of the corresponding pulse. By moving a mirror mounted on a translation stage we could select and analyze different spatial parts of the beam. In addition, a standard CCD camera with an infrared filter (long-pass filter at approximately $700 \mathrm{~nm}$ ) coupled to a frame-grabber was used to register the spatial distribution 
of the beam transmitted through the sample. For the two cases mentioned above we performed measurements with different input pulse energies starting with a very low energy at which nonlinear effects can be neglected up to the energy of about $2 \mu \mathrm{J}$ which corresponds roughly to the threshold of white light continuum generation in the sample.

\section{The experimental results}

We have found that the results of the experiment are significantly different for the two cases studied here, i.e. "large beam" and "small beam".

For the "large beam" case (120 $\mu \mathrm{m}$ focus diameter) which is similar to what has been studied by others before $[16,17]$ we observe very little variation in the transmitted beam shape even for high input pulse energy when significant spectral and temporal shaping is observed. The $\omega-k_{\perp}$ maps for three different input pulse energies are shown in Fig. 4. For the lowest energy the map shows a smooth distribution - both the frequency and wave-vector spectra display no significant structure. For higher pulse energies the frequency spectrum acquires significant structure. Besides a fast modulation around the peak of the spectrum we observe the beginning of spectral splitting. The splitting is very well pronounced for the
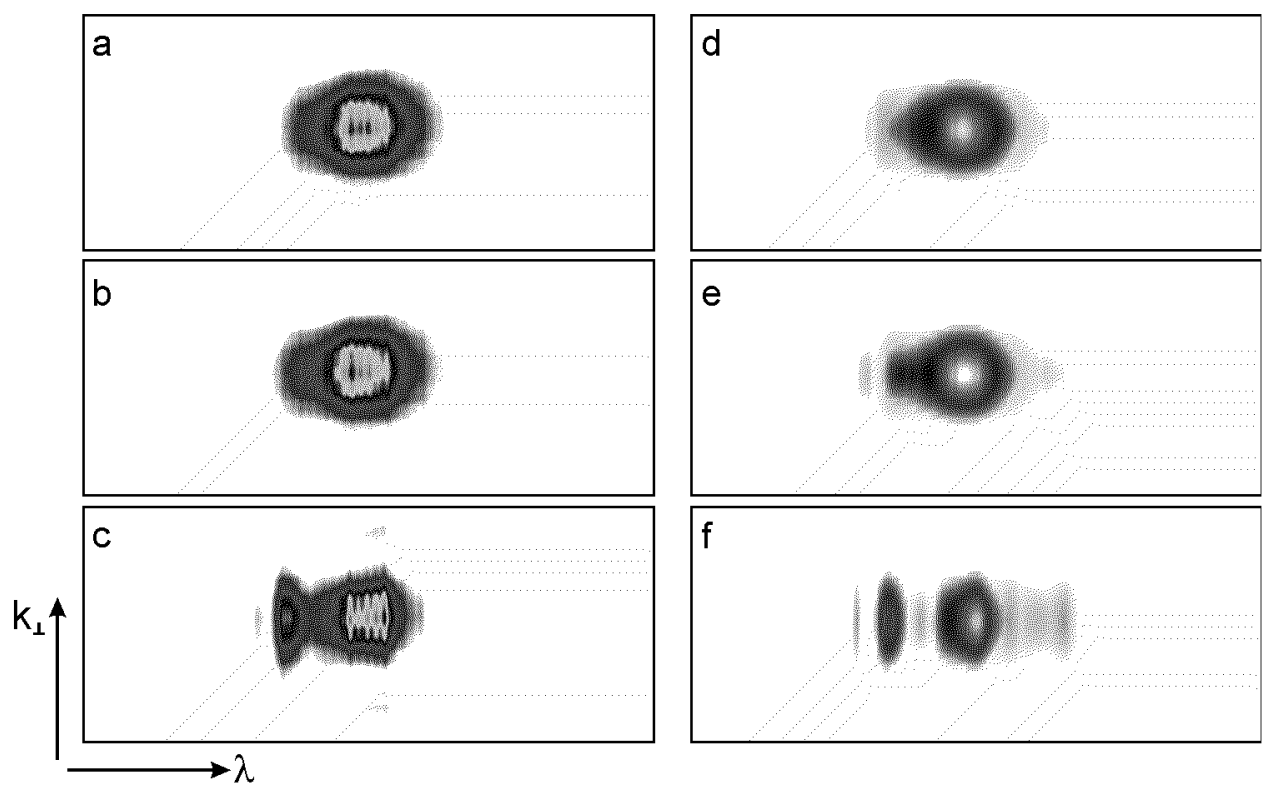

Fig. 4. The $\omega-k_{\perp}$ map for the "large beam" case. (a)-(c) Experimental data for the input pulse energy $0.1 \mu \mathrm{J}, 1 \mu \mathrm{J}$, and $2 \mu \mathrm{J}$. (d)-(f) Numerical modeling results for the corresponding input pulse energies. Spectral range is $740-860 \mathrm{~nm}$. 

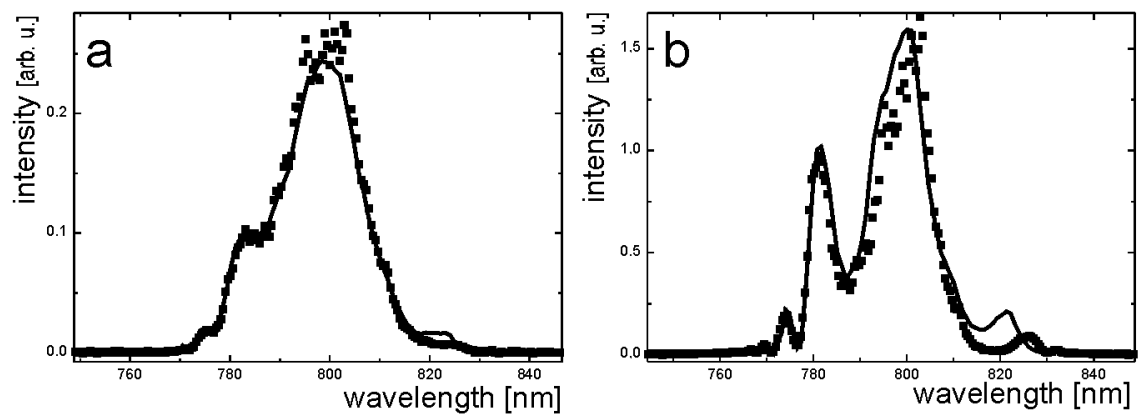

Fig. 5. Comparison between the experimental (squares) and theoretical (line) on-axis spectra for two different input pulse energies: $0.3 \mu \mathrm{J}$ (a) and $0.8 \mu \mathrm{J}$ (b).

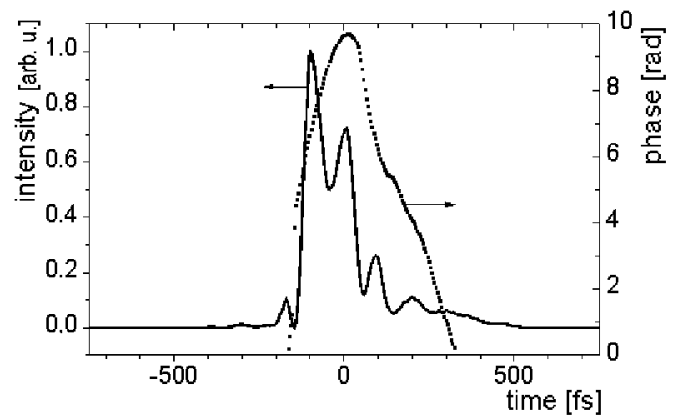

Fig. 6. An example of the temporal dependence of amplitude (line) and phase (dots) of the pulse in the "large beam" case.

highest energy studied. In addition to the two main maxima there are also smaller ones at higher and lower frequencies. We do not observe any dramatic changes in the beam shape - there is only a slight narrowing of the distribution in the $k_{\perp}$ direction. What is even more important, the frequency spectrum does not depend on the $k_{\perp}$ vector. Therefore, to describe the beam properties it is sufficient to measure it on the axis. The results of such measurements are shown in Figs. 5 and 6 . Figure 5 presents the pulse spectrum for two input energies. Spectral splitting is clearly visible. It is worth mentioning that the effect is very reproducible. The results of FROG measurements of the central part of the beam are shown in Fig. 6. The pulse shows a distinct splitting in time domain. The subpulses are not completely separated but, as they have different spectral content (which can be deduced from the retrieved temporal phase), they would move apart from each other if propagated further in silica.

The experimental results are quite different for the "small beam" case $(20 \mu \mathrm{m}$ focus diameter). When the input pulse energy is increased we observe very interesting changes in the beam profile. This is illustrated in Fig. 7 which shows images of the transmitted beam profile taken with the CCD camera. The first 

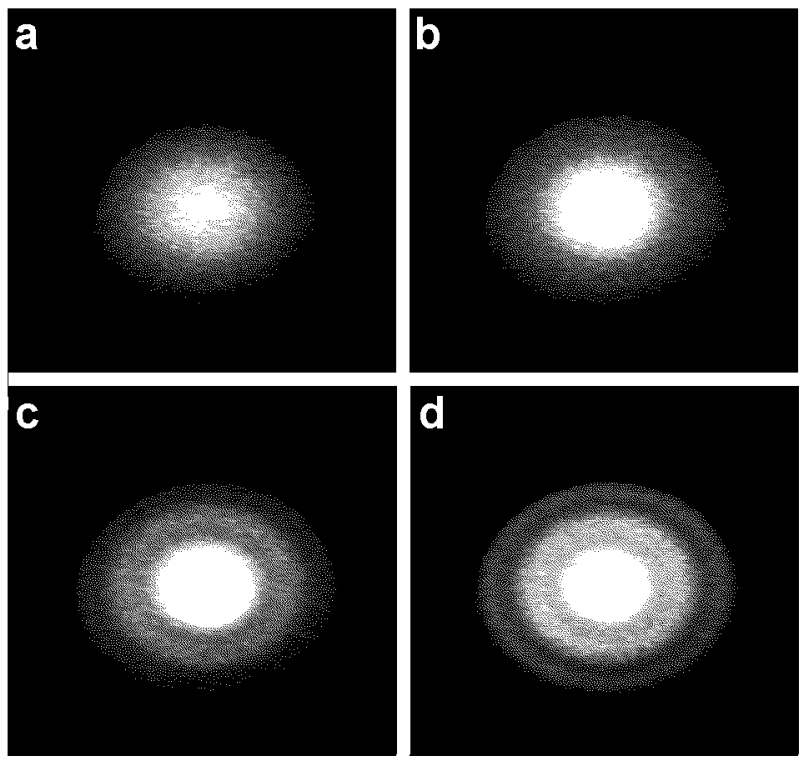

Fig. 7. The far field intensity distribution in the beam transmitted through the sample in the "small beam" case. The energy of the input pulse is $0.3 \mu \mathrm{J}(\mathrm{a}), 0.6 \mu \mathrm{J}(\mathrm{b}), 0.8 \mu \mathrm{J}$ (c), and $0.9 \mu \mathrm{J}(\mathrm{d})$.

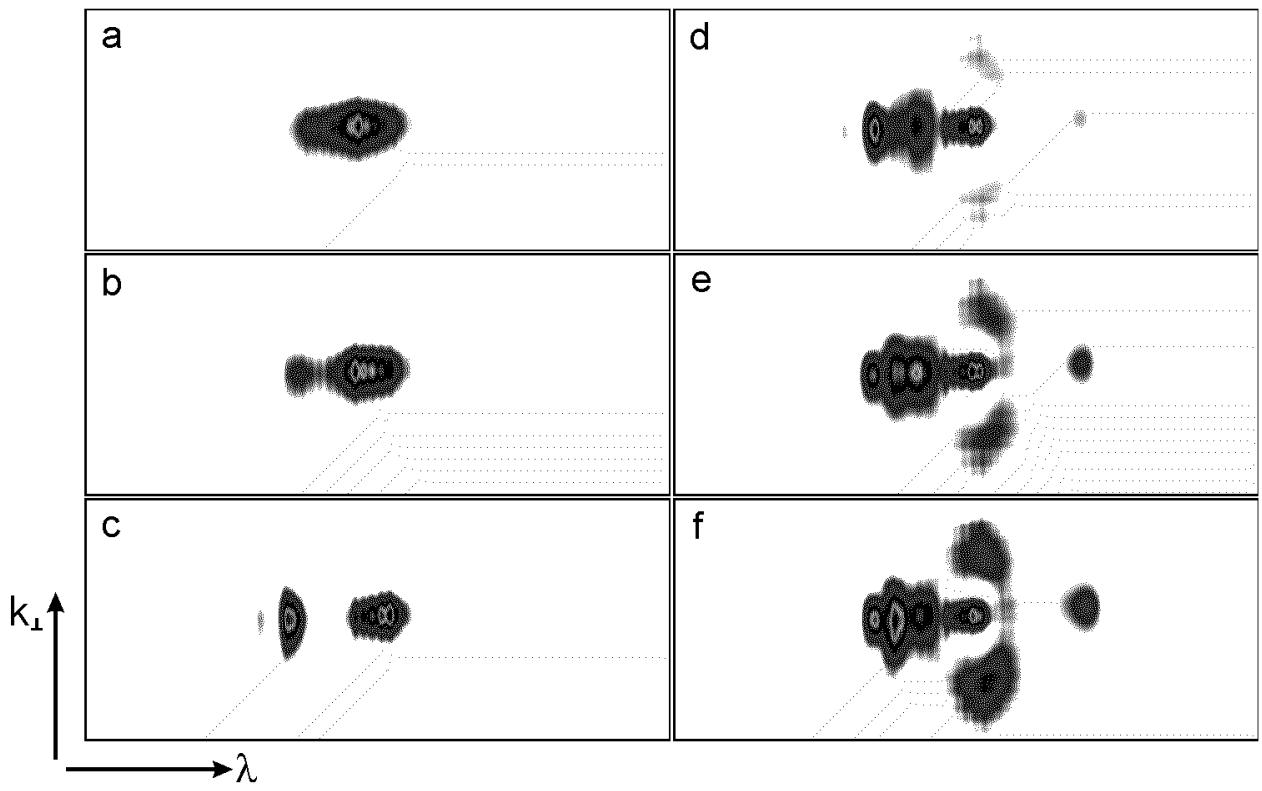

Fig. 8. The $\omega-k_{\perp}$ map for the "small beam" case. The energy of the input pulse was: (a) $0.1 \mu \mathrm{J}$, (b) $0.4 \mu \mathrm{J}$, (c) $0.6 \mu \mathrm{J}$, (d) $0.8 \mu \mathrm{J}$, (e) $1.0 \mu \mathrm{J}$, (f) $1.1 \mu \mathrm{J}$. Spectral range is $740-860 \mathrm{~nm}$. 
image corresponds to a very small input energy at which the beam profile does not experience any significant changes. When the input pulse energy is increased, the beam starts to self-focus and for still higher energies well-defined rings appear. The ring structure varies with the input energy and the general rule is that the higher the energy the richer the ring structure. It should be mentioned that for higher energies corresponding to a well-developed ring structure the spectral broadening of the pulse is quite large and one can easily see a visible beam with a naked eye. An additional slight increase in the input pulse energy leads to a strong visible radiation - white light continuum. The $\omega-k_{\perp}$ maps for this case are shown in Fig. 8. Similar to the "large beam" case, when the input energy is increased, initially, we observe spectral splitting. For the highest energy studied here there are at least five well defined and separated peaks in the transmitted pulse spectrum. However, for higher energies an additional effect comes into play. Separate islands away from the $k_{\perp}=0$ line appear. Those islands correspond to the rings observed with the CCD camera. The structure of the $\omega-k_{\perp}$ maps is quite complex. In particular, it is easy to notice that the spectral content of the rings is different from the main part of the beam centered around $k_{\perp}=0$. The spectrum of the rings is quite narrow when compared with the spectrum in the beam center. Also the spectrum of the rings is centered around the maximum of the input pulse spectrum.

\section{Discussion}

We have modeled the propagation of laser pulses in fused silica sample using the numerical procedure described in Sec. 2. The input data for the procedure has to include full information on both temporal and spatial properties of the input pulse. As an input to the procedure we have used the electric field of the laser pulse measured with the FROG apparatus. We have also assumed that the spatial distribution of the field in the input plane of the sample results from imaging (with proper magnification) of the pinhole. The numerical procedure allows us to follow the pulse evolution inside the sample $E(x, t)$. It also provides the $\omega-k_{\perp}$ distribution which can be directly compared with the $\omega-k_{\perp}$ maps measured in the experiment. Such a comparison for the "large beam" case is shown in Fig. 4. The agreement between the theory and experiment is quite good. In both cases we observe spectral splitting — an initially smooth spectrum breaks into two main and several weaker bands as the input pulse energy is increased. Also, in agreement with the experiment, the numerical results do not show any significant spatial pulse break-up. The quality of the agreement can be better assessed with the help of Fig. 5 which shows measured and calculated spectra of the pulse for two input energies. Both spectra were taken on the beam axis. Our model correctly predicts splitting of the pulse spectrum into several bands and gives an almost perfect fit on short wavelength side. The agreement is worse on the long wavelength side where 
the experimentally obtained peaks are shifted to the red with respect to the peaks predicted by the model. One possible explanation for this effect might be Raman scattering which is not included in our model. The results of the calculations (not shown here) for the "small beam" case show only a qualitative agreement with experiment. There are several spectral bands appearing on the beam axis with positions and amplitudes similar to those observed in the experiment (see Fig. 8). What is even more important, similar to the experiment, we observe development of rings at higher input energies. Those rings have spectrum which is completely different from the spectrum on the beam axis. Thus the model predicts most of the features observed in the experiment. However, the qualitative agreement is not very good. This is probably partly due to numerical effects. A very rich structure that develops in the pulse in this case calls for using large and finely spaced grids in both time and frequency domains. We could not do this because of our limited computing capabilities. We are currently working on developing a more efficient code that, we hope, will handle the calculations better.

\section{Conclusions}

We have observed and partially analyzed some aspects of nonlinear propagation of femtosecond laser pulses in isotropic, transparent, nonlinear media. When the power of a tightly focused input pulse is higher than the critical power for self-focusing both temporal and spatial pulse splitting is observed. The rings have completely different spectra than the main part of the pulse propagating along the beam axis. This is, to our knowledge, the first report on pulse spatial break-up after nonlinear propagation in a transparent Kerr medium. All the earlier measurements were limited to the beam axis. Using the numerical model for the pulse propagation we can partially explain formation of the rings although quantitative agreement is still not satisfactory. In the future we plan to develop a more efficient numerical code to perform the calculations with sufficient accuracy and to use SPIDER [33] instead of FROG to characterize the output pulses. This should allow us to completely characterize the pulse across the beam profile.

\section{Acknowledgments}

This work was partially supported by the State Committee for Scientific Research in particular under grant no. 2P03B 019 18. Part of calculations were carried out at the Interdisciplinary Center for Mathematical and Computational Modeling (ICM), Warsaw University. 


\section{References}

[1] J. Reichert, M. Niering, R. Holzwarth, M. Weitz, Th. Udem, T.W. Hansch, Phys. Rev. Lett. 84, 3232 (2000).

[2] S.A. Diddams, D.J. Jones, J. Ye, S.T. Cundiff, J.L. Hall, J.K. Ranka, R.S. Windeler, R. Holzwarth, T. Udem, T.W. Hansch, Phys. Rev. Lett. 84, 5102 (2000).

[3] K.B. Moller, A.H. Zewail, Chem. Phys. Lett. 295, 1 (1998).

[4] P.Y. Cheng, D. Zhong, A.H. Zewail, J. Chem. Phys. 105, 6216 (1996).

[5] M. Dantus, R.M. Bowman, M. Gruebele, A.H. Zewail, J. Chem. Phys. 91, 7437 (1989).

[6] S. Savikhin, Z. Wenli, R.E. Blankenship, W.S. Struve, Biophys. J. 66, 110 (1994).

[7] A.V. Sharkov, I.V. Kryukov, E.V. Khoroshilov, P.G. Kryukov, R. Fisher, H. Sheer, T. Gillbro, Chem. Phys. Lett. 191, 633 (1992).

[8] S. Hess, F. Feldchtein, A. Babin, I. Nurgaleev, T. Pullerits, A. Sergeev, V. Sundstrom, Chem. Phys. Lett. 216, 247 (1993).

[9] C.K. Choi, Y.H. Kwon, J.S. Krasinski, G.H. Park, G. Setlur, J.J. Song, Y.C. Chang, Phys. Rev. B 63, 115315 (2001).

[10] D.F. Underwood, T. Kippeny, S.J. Rosenthal, J. Phys. Chem. B 105, 436 (2001).

[11] A. Othonos, J. Appl. Phys. 83, 1789 (1998).

[12] M.D. Perry, G. Mourou, Science 264, 917 (1994).

[13] C. Radzewicz, M.J. la Grone, J.S. Krasinski, Opt. Commun. 126, 185 (1996).

[14] C. Radzewicz, J.S. Krasinski, M.J. la Grone, M. Trippenbach, Y.B. Band, J. Opt. Soc. Am. B 14, 420 (1997).

[15] J.K. Ranka, R.W. Schirmer, A.L. Gaeta, Phys. Rev. Lett. 77, 3783 (1996).

[16] S.A. Diddams, H.K. Eaton, A.A. Zozulya, T.S. Clement, Opt. Lett. 23, 379 (1998).

[17] A.A. Zozulya, A.G. Van Engen, T.S. Clement, Phys. Rev. Lett. 82, 1430 (1999).

[18] Y.B. Band, M. Trippenbach, Phys. Rev. Lett. 76, 1457 (1996).

[19] M. Trippenbach, Y.B. Band, J. Opt. Soc. Am. B 13, 1403 (1996).

[20] M. Trippenbach, T.C. Scott, Y.B. Band, Opt. Lett. 22, 5791 (1997).

[21] J.A. Fleck, J.R. Morris, M.D. Feit, Appl. Opt. 10, 129 (1976); M.D. Feit, J.A. Fleck, Appl. Opt. 17, 3390 (1978); Appl. Opt. 18, 2843 (1979).

[22] P. Chernev, V. Petrov, Opt. Lett. 17, 172 (1992).

[23] J.E. Rothenberg, Opt. Lett. 17, 583 (1992).

[24] G.C. Luther, A.C. Newell, J.V. Maloney, E.M. Wright, Opt. Lett. 19, 789 (1994); 19, 862 (1994).

[25] M. Trippenbach, Y.B. Band, Phys. Rev. A 56, 4242 (1997).

[26] M. Trippenbach, Y.B. Band, Phys. Rev. A 57, 4791 (1998).

[27] G. Fibich, G. Papanicolaou, Phys. Lett. A 239, 167 (1998).

[28] G. Fibich, Phys. Rev. Lett. 76, 4356 (1996).

[29] M. Trippenbach, W. Wasilewski, G.M.W. Bryant, G. Fibich, Y.B. Band, to be published. 
[30] G.P. Agrawal, Nonlinear Fiber Optics, Academic Press, Boston 1989.

[31] D.J. Kane, R. Trebino, IEEE J. Quantum Electron. QE-29, 571 (1993).

[32] K.W. DeLong, R. Trebino, J. Opt. Soc. Am. A 11, 2429 (1994).

[33] C. Iaconis, I.A. Walmsley, IEEE J. Quantum Electron. QE-35, 501 (1999). 